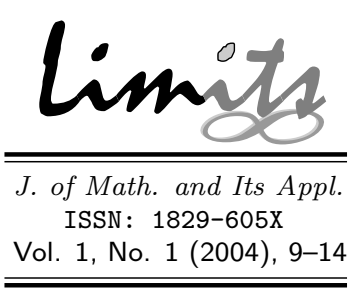

\title{
Martingales and Financial Mathematics
}

\author{
J.A.M. van der Weide \\ Delft University of Technology \\ j.a.m.vanderweide@its.tudelft.nl
}

\begin{abstract}
In this expository paper, we will discuss the role played by martingales in Financial Mathematics. More precisely, we will restrict ourselves to a mathematical formulation of the economical concept of an arbitrage-free, complete market and the pricing of derivatives in such models. For a clear exposition, we only consider the discrete case. We also discuss the Cox-Ross-Rubinstein model which is still one of the most used models in Finance.
\end{abstract}

\section{Introduction}

Consider the following example. On a market, shares of a certain stock ${ }^{1)}$ are traded for a price of 10 Euro. At the end of the year the price will have gone up to 15 Euro or down to 5 Euro. We want to enter into the following agreement (contract): if the price has gone down, then we will get 5 Euro at the end of the year, if the price has gone up we will receive no money at the end of the year. The time at which the money will be paid is also called the maturity time of the contract. Assume that this contract is also traded on the market. What is a fair price for this contract?

Using classical probability theory we could argue as follows: the expected value of the amount of money that we will receive at the end of the year equals $5 \times$ the probability that the price will go down. So, if we estimate this probability as being 0.2 , the price for the contract will be 1 Euro.

But assume that it is possible to borrow money from a bank now that has to be paid back at the end of the year and that the interest rate $R=0.1$. One can buy one share of the stock and two contracts using 12 Euro, that has been borrowed from the bank. So the total investment now is 0 . At the end of the year, we will sell the stock, possibly get money from the contracts and pay back our loan. What will happen at the end of the year? If the price has gone up, we sell the stock for 15 Euro and we pay our loan back, so we end up with $15-12 \times(1+0.1)=1.8$ Euro. On the other hand, if the price of the stock has gone down, we get 5 Euro for the stock and 10 Euro from the two contracts. Paying our loan back, we end up again with 1.8 Euro. So with an investment 0 , we get always 1.8 Euro at the end of the year. So many people will use this opportunity and, in a free market economy, the price of the contract will rise and this opportunity to get risk-free money will disappear. In other words, the price of 1 Euro is not correct and is actually too low.

To calculate the correct price, we will proceed as follows: buy a number $\phi_{1}$ of shares and put an amount of $\phi_{0}$ Euro in the bank, the vector $\Phi=\left(\phi_{0}, \phi_{1}\right)$ is called a portfolio. At this point we make some assumptions. It is possible to buy arbitrary parts of a share of the stock, also a negative number of shares is allowed: a negative number of shares means shorting the stock ${ }^{2)}$. So $\Phi$ is a vector $\mathbb{R}^{2}$. By an easy calculation, one finds that the portfolio $\Phi=(75 / 11,-1 / 2)$ has the same value at the end of the year as what we will receive from 
the contract. This portfolio is called a replicating portfolio for the claim, i.e. the amount of money that an owner of the agreement earns at maturity (the end of the year). So the price for the contract is the money needed to set up the replicating portfolio, i.e. 20/11 Euro. If the price of the contract would be less than $20 / 11$, one could sell short the replicating portfolio and use the money for buying the contract. The (positive) difference is put on the bank account and the contract will furnish the money needed to return the replicating portfolio. In the same way one constructs a strategy with a risk-free return in the case the price of the contract is higher than 20/11. Strategies with initial investment 0 and a risk-free (positive) return are called arbitrage opportunities. It is an economical principle that there exist no arbitrage opportunities for markets in equilibrium. Economists call a market complete if there exists a replicating portfolio for every claim. In their papers [1] and [2] dating from around 1980, Harrison, Kreps and Pliska showed that a model for an economy does not allow arbitrage opportunities if and only if their exist an equivalent martingale measure. Secondly, a model without arbitrage opportunities is complete if and only if there is a unique equivalent martingale measure. In Section 2 we will explain what martingales are. In Section 3 we discuss the role of martingales in Financial Mathematics.

\section{$2 \quad$ Martingales}

Let $\Omega=\left\{\omega_{1}, \ldots, \omega_{K}\right\}$ be a finite set and $P$ a probability measure on $\Omega$ such that $P\left(\omega_{k}\right)>$ $0, k=1, \ldots, K$. Let $\mathcal{F}$ be an algebra ${ }^{3)}$ of subsets of $\Omega$ generated by the partition $\mathcal{P}$, i.e. every set in $\mathcal{F}$ can be written in a unique way as a union of elements of $\mathcal{P}$. Note that the partition element containing $\omega$ is equal to the intersection of all sets in $\mathcal{F}$ containing $\omega$. Let $\mathcal{P}=\left\{B_{1}, \ldots, B_{n}\right\}$ and $Y: \Omega \mapsto \mathbb{R}$ a random variable. The conditional expectation of $Y$ given that the event $B$ has occurred is defined by $\left.{ }^{4}\right)$

$$
\begin{aligned}
E(Y \mid B) & =\sum_{\omega} Y(\omega) P(\omega \mid B) \\
& =\frac{1}{P(B)} E\left(Y 1_{B}\right) .
\end{aligned}
$$

The conditional expectation of $Y$ given the information in the algebra $\mathcal{F}$ is the random vari- able $E(Y \mid \mathcal{F})$ defined by:

$$
E(Y \mid \mathcal{F})(\omega)=\sum_{i=1}^{n} E\left(Y \mid B_{i}\right) \cdot 1_{B_{i}}(\omega) .
$$

So $E(Y \mid \mathcal{F})$ is a $\mathcal{F}$-measurable random variable ${ }^{5)}$.

Theorem 2.1 Let $\mathcal{F}$ be an algebra of subsets of $\Omega$. Then $E(Y \mid \mathcal{F})$ is the unique, $\mathcal{F}$ measurable random variable such that for all $F \in \mathcal{F}:$

$$
E\left[1_{F} E(Y \mid \mathcal{F})\right]=E\left[1_{F} Y\right]
$$

It is sufficient that (3) is satisfied for all sets $B$ in the partition $\mathcal{P}$ generating $\mathcal{F}$.

Proof : First, let $B \in \mathcal{P}$. By formula (2) and (1) :

$$
\begin{aligned}
E\left[1_{B} E(Y \mid \mathcal{F})\right] & =E\left[1_{B} E(Y \mid B)\right] \\
& =E(Y \mid B) P(B) \\
& =E\left[1_{B} Y\right] .
\end{aligned}
$$

For arbitrary $F \in \mathcal{F},(3)$ follows from the linearity of the expectation, since $F$ can be written as a union of partition elements.

To prove the uniqueness, let $X$ be an $\mathcal{F}$-measurable random variable such that $E\left[1_{F} X\right]=E\left[1_{F} Y\right]$ for every $F \in \mathcal{F}$. Let $\omega \in \Omega$ and let $B$ be the partition element containing $\omega$. . Since $X$ is constant on $B$, it follows, by taking $F=B$ that

$$
X(\omega) P(B)=E\left[1_{B} Y\right],
$$

and $X(\omega)=E(Y \mid \mathcal{F})(\omega)$.

It follows from Theorem (2.1) that we can define the conditional expectation $E(Y \mid \mathcal{F})$ as the $\mathcal{F}$-measurable random variable satisfying (3). Actually, this definition is used in the general case where $\Omega$ is not finite. From Theorem (2.1) we can easily derive the following properties of conditional expectations:

- if $X$ is an $\mathcal{F}$-measurable random variable, then

$$
E(X Y \mid \mathcal{F})=X E(Y \mid \mathcal{F})
$$

- for any $\mathcal{F}$-measurable random variable $X$, we have

$$
E[X E(Y \mid \mathcal{F})]=E[X Y]
$$


- (tower-property) for any two algebra's $\mathcal{F}_{1}, \mathcal{F}_{2}$, such that $\mathcal{F}_{1} \subset \mathcal{F}_{2}$ we have

$$
\begin{aligned}
& E\left(E\left(Y \mid \mathcal{F}_{1}\right) \mid \mathcal{F}_{2}\right) \\
& \quad=E\left(E\left(Y \mid \mathcal{F}_{2}\right) \mid \mathcal{F}_{1}\right) \\
& \quad=E\left(Y \mid \mathcal{F}_{1}\right) .
\end{aligned}
$$

- if $Y$ and $\mathcal{F}$ are independent ${ }^{6)}$, then

$$
E(Y \mid \mathcal{F})=E Y \text {. }
$$

Let $Y_{0}, Y_{1}, \ldots, Y_{T}$ be a sequence of random variables defined on the sample space $\Omega$. Such a sequence is also called a stochastic process, the indices $0,1, \ldots, T$ are usually interpreted as time epochs. A filtration is an increasing sequence $\mathcal{F}_{0} \subset \mathcal{F}_{1} \subset \cdots \subset \mathcal{F}_{T}$ of algebra's of subsets of $\Omega$. The algebra $\mathcal{F}_{i}$ represents the information available at time $i$; it contains all events of which it is known at time $i$ whether they happened or not. Usually we take $\mathcal{F}_{0}=\{\emptyset, \Omega\}$. The stochastic process $\left(Y_{t} ; t=0,1, \ldots, T\right)$ is said to be adapted to the filtration $\left\{\mathcal{F}_{t}\right\}$ (or $\left\{\mathcal{F}_{t}\right\}$-adapted) if $Y_{t}$ is $\mathcal{F}_{t}$-measurable, $t=0,1, \ldots, T$. So, this means that for an adapted process $Y_{t}$ is known at time $t$. As an example of a filtration, we define $\mathcal{F}_{t}$ as the algebra generated by the partition $\left\{Y_{0}=y_{0}, Y_{1}=y_{1}, \ldots, Y_{t}=y_{t}\right\}$ where $y_{s}$ is a possible value for $Y_{s}$. This filtration is the smallest filtration such that the process $\left(Y_{t}\right)$ is adapted and is also called the natural filtration of the process $\left(Y_{t}\right)$.

A martingale with respect to the filtration $\left\{\mathcal{F}_{t}\right\}$ is defined as an $\left\{\mathcal{F}_{t}\right\}$-adapted process $\left(M_{t}\right)$ with the property

$$
E\left(Y_{t} \mid \mathcal{F}_{t-1}\right)=Y_{t-1}, \quad t=1, \ldots, T .
$$

It follows from the tower property of conditional expectations that for a martingale

$$
E\left(Y_{T} \mid \mathcal{F}_{t}\right)=Y_{t}, \quad t=1, \ldots, T .
$$

A well-known example of a martingale is the fraction of black balls in Polya's urn ${ }^{7)}$.

\section{Applications in Financial Mathematics}

We will consider a finite market model that is specified by

- a finite set $\{0,1, \ldots, T\}$ of dates at which trading or consumption is possible;
- a finite sample space $\Omega=\left\{\omega_{1}, \ldots, \omega_{K}\right\}$ whose elements represent the possible states of the world;

- a probability measure $\mathbb{P}$ on $\Omega$ with $\mathbb{P}\left(\omega_{k}\right)=p_{k}>0, k=1, \ldots, K ;$

- a filtration $\left(\mathcal{F}_{t}\right)_{0 \leq t \leq T}$, where $\mathcal{F}_{t}$ represents the information that is known to the investors at time $t, \mathcal{F}_{0}=\{\emptyset, \Omega\}$ and $\mathcal{F}_{T}$ is the collection of all subsets of $\Omega$;

- a bank account process $B=\left(B_{t} ; 0 \leq t \leq\right.$ $T)$, with $B_{t}=(1+R)^{t}$, the value of a savings account at time $t$ when 1 Euro is deposited at time 0 ;

- a stock with price process $S=\left(S_{t} ; 0 \leq\right.$ $t \leq T)$, adapted to the filtration $\left(\mathcal{F}_{t}\right)$.

Denote, for $t=1, \ldots, T$, by $\Phi(t)=$ $\left(\phi_{0}(t), \phi_{1}(t)\right)$ the portfolio held during the time interval $[t-1, t)$, where $\phi_{0}(t) B_{t-1}$ is the amount of money at the bank and $\phi_{1}$ the number of shares of the stock. A trading strategy $\Phi=$ $(\Phi(t) ; 1 \leq t \leq T)$ is defined as a collection of portfolio's $\Phi(t): \Omega \mapsto \mathbb{R}^{2}$ such that $\Phi(t)$ is $\mathcal{F}_{t-1}$-measurable. This means that we set up at time 0 the portfolio $\Phi(1)$. At time 1 we sell the portfolio $\Phi(1)$ and buy the portfolio $\Phi(2)$ for the prices at time 1 . In general, $\Phi(2)$ will depend on the state of the world as known to us at time 1 , or, in other words, $\Phi(2)$ is constant on the elements of the partition generating $\mathcal{F}_{1}$ or, in other words, $\Phi(2)$ is $\mathcal{F}_{1}$-measurable. And so forth. A portfolio is self-financing if the money that we get from selling $\Phi(t)$ at time $t$ equals the money that we need to buy $\Phi(t+1)$ :

$$
\begin{aligned}
& \phi_{0}(t) B_{t}+\phi_{1}(t) S_{t} \\
& \quad=\phi_{0}(t+1) B_{t}+\phi_{1}(t+1) S_{t} .
\end{aligned}
$$

For a self-financing trading strategy $\Phi$ we define the value at time $t$ by:

$$
V_{t}(\Phi)= \begin{cases}\phi_{0}(1) B_{0}+\phi_{1}(1) S_{0} & \text { if } t=0 \\ \phi_{0}(t) B_{t}+\phi_{1}(t) S_{t} & \text { if } t \geq 1\end{cases}
$$

An arbitrage opportunity is a self-financing trading strategy $\Phi$ with $V_{0}(\Phi)=0$ and $V_{T}(\Phi) \geq 0$ and at least for one state $\omega$ of the world $V_{T}(\Phi)(\omega)>0$. This means that an arbitrage opportunity is a strategy where we can get money from an initial investment $=0$ without any risk to loose money. It is an economical principle that in an equilibrium situation arbitrage opportunities do not exist. In order 
to give a mathematical description for market models that do not allow arbitrage opportunities, we introduce the concept of an equivalent martingale measure.

Definition 3.1 A probability measure $Q$ on $\Omega$ is called an equivalent martingale measure if

(a) $Q(\omega)>0$ for every $\omega \in \Omega$, and

(b) the discounted price process $\left(\tilde{S}_{t}\right)$, defined by

$$
\tilde{S}_{t}=\frac{S_{t}}{B_{t}} \quad t=0,1, \ldots, T
$$

is a martingale with respect to the filtration $\left(\mathcal{F}_{t}\right)$ and the probability measure $Q$.

If $Q$ is an equivalent martingale measure, then the discounted value process $\left(\tilde{V}_{t}(\Phi)\right)=$ $\left(V_{t}(\Phi) / B_{t}\right)$ of a self-financing trading strategy is a martingale. It is clear that the process $\left(\tilde{V}_{t}(\Phi)\right)$ is $\left\{\mathcal{F}_{t}\right\}$-adapted. Further

$$
\begin{aligned}
E_{Q}\left(\tilde{V}_{t}(\Phi) \mid \mathcal{F}_{t-1}\right) \\
\quad=E_{Q}\left(\phi_{0}(t)+\phi_{1}(t) \tilde{S}_{t} \mid \mathcal{F}_{t-1}\right) \\
\quad=\phi_{0}(t)+\phi_{1}(t) E_{Q}\left(\tilde{S}_{t} \mid \mathcal{F}_{t-1}\right) \\
\quad=\phi_{0}(t)+\phi_{1}(t) \tilde{S}_{t-1} \\
\quad=\left(\phi_{0}(t) B_{t-1}+\phi_{1}(t) S_{t-1}\right) / B_{t-1}
\end{aligned}
$$

where we have used that $\phi_{0}(t)$ and $\phi_{1}(t)$ are $\mathcal{F}_{t-1}$-measurable. Now, $\phi_{0}(t) B_{t-1}+$ $\phi_{1}(t) S_{t-1}=V_{t-1}(\Phi)$, since $\Phi$ is self-financing and this implies the martingale property of the discounted value process.

The following Theorem gives a mathematical formulation of an arbitrage-free market model.

Theorem 3.2 A market model is free from arbitrage opportunities if and only if there exists an equivalent martingale measure.

A notion important for the pricing of derivatives, is completeness. A claim is a random variable $X$ representing the pay-out at time $T$ of some contract. For example, the owner of a European call option with strike price $K$ and time of maturity $T$ has the right (not the obligation) to buy a share of the stock for a price $K$ at time $T$. So, if at time $T$ the price $S_{T}>K$, he can buy the stock for $K$ and sell for $S_{T}$ giving a pay-out $S_{T}-K$. If $S_{T} \leq K$ then the option is not exercised, i.e. the owner does not use his right to buy a stock for price $K$. In this case $X=\max \left(S_{T}-K, 0\right)$. Another example is the
Asian strike call option which is a European option with strike price equal to the average stock price over $[0, T]$. The pay-out of an Asian strike call is $X=\max \left(S_{T}\right.$-average stock price, 0$)$. A claim is attainable or can be replicated if there exists a self-financing strategy $\Phi$ such that the value of the strategy at time $T$ is equal to $X$ : $V_{T}(\Phi)=X$. In this case $\Phi$ is also called a replicating strategy or hedging strategy for claim $X$.

If the market is arbitrage-free, there exists an equivalent martingale measure $Q$ by Theorem (3.2.) If $X$ is an attainable claim and if $\Phi=(\Phi(t))$ is a replicating trading strategy, then $V_{0}(\Phi)$, the money needed to set up the strategy $\Phi$, is a fair price for claim $X$. Since the discounted value process of a self-financing trading strategy is a martingale, it follows that

$$
\tilde{V}_{0}(\Phi)=E_{Q}\left(\tilde{V}_{T}(\Phi) \mid \mathcal{F}_{0}\right) .
$$

Now, $\tilde{V}_{0}(\Phi)=V_{0}(\Phi)$ and

$$
\begin{aligned}
& E_{Q}\left(\tilde{V}_{T}(\Phi) \mid \mathcal{F}_{0}\right) \\
& \quad=\frac{1}{(1+R)^{T}} E_{Q}\left(V_{T}(\Phi) \mid \mathcal{F}_{0}\right) \\
& \quad=\frac{1}{(1+R)^{T}} E_{Q}(X) .
\end{aligned}
$$

So a fair price for claim $X$ can be represented as

$$
V_{0}(\Phi)=\frac{1}{(1+R)^{T}} E_{Q}(X) .
$$

It follows also that for another replicating strategy $\Psi$ the fair price $V_{0}(\Psi)=V_{0}(\Phi)$. There could be different fair prices if there are more than one equivalent martingale measures.

An arbitrage-free market model is complete if every claim is attainable. In the following Theorem we give a mathematical formulation of this property.

Theorem 3.3 An arbitrage-free market model is complete if and only if there exists only one equivalent martingale measure.

For proofs of Theorem (3.2) and (3.3) we refer to reference [3] or [4].

We continue with an important example.

Example Consider the one period model with trading dates 0,1 and with two states of the world: $\Omega=\left\{\omega_{1}, \omega_{2}\right\}$. The filtration is given by $\mathcal{F}_{0}=\{\emptyset, \Omega\}$ and $\mathcal{F}_{1}=\left\{\emptyset,\left\{\omega_{1}\right\},\left\{\omega_{2}\right\}, \Omega\right\}$. The price process is given by $S_{0}=s, S_{1}\left(\omega_{1}\right)=$ $u s$ and $S_{1}\left(\omega_{2}\right)=d s$, where $0<d<u$. 
An equivalent martingale measure $Q$ has to satisfy the equation

$$
E_{Q}\left(\tilde{S}_{1} \mid \mathcal{F}_{0}\right)=\tilde{S}_{0}
$$

It follows easily that this equation is equivalent with

$$
u Q\left(\omega_{1}\right)+d Q\left(\omega_{2}\right)=1+R
$$

with solution $Q\left(\omega_{1}\right)=1-Q\left(\omega_{2}\right)=q_{u}$, where

$$
q_{u}=\frac{(1+R)-d}{u-d}
$$

So $Q$ is an equivalent martingale measure if and only if $d<1+R<u$. So, if this condition is satisfied, the model is arbitrage-free and complete. The replicating strategy for a claim $X$ is given by

$$
\phi_{0}=\frac{1}{1+R} \frac{x_{2} u-x_{1} d}{u-d}
$$

and

$$
\phi_{0}=\frac{1}{s} \frac{x_{1}-x_{2}}{u-d}
$$

where $x_{i}=X\left(\omega_{i}\right), i=1,2$.

We finish with the Cox-Ross-Rubinstein model. The trading dates are now $0,1, \ldots, T$ and the sample space $\Omega=\{u, d\}^{T}$, the set of all sequences $\omega=\left(\omega_{1}, \ldots, \omega_{T}\right)$ of length $T$ consisting of $u$ 's and $d$ 's. To make the notation more simple we introduce the random variables $Z_{i}, i=1, \ldots T$ defined by

$$
Z_{i}(\omega)= \begin{cases}0 & \text { if } \omega_{i}=d \\ 1 & \text { if } \omega_{i}=u\end{cases}
$$

Let $U_{t}=Z_{1}+\ldots+Z_{t}$ for $t=1, \ldots, T$. then the price process of the stock is given by $S_{0}=s$ and

$$
S_{t}(\omega)=s u^{U_{t}(\omega} d^{t-U_{t}(\omega)}
$$

Note that

$$
S_{t+1}=S_{t} u^{Z_{t+1}} d^{1-Z_{t+1}} .
$$

The algebra's $\mathcal{F}_{t}$ are generated by the partitions $\left\{Z_{1}=a_{1}, \ldots, Z_{t}=a_{t}\right\}$ where $\left(a_{1}, \ldots, a_{t}\right) \in\{0,1\}^{t}$. Note that the price process is adapted to this filtration, it is even the natural filtration of the price process.

A necessary condition for an equivalent martingale measure $Q$ is that

$$
E_{Q}\left(\tilde{S}_{t+1} \mid \mathcal{F}_{t}\right)=\tilde{S}_{t}
$$

We have

$$
\begin{aligned}
& E_{Q}\left(\tilde{S}_{t+1} \mid \mathcal{F}_{t}\right)=E_{Q}\left(\frac{S_{t+1}}{B_{t+1}} \mid \mathcal{F}_{t}\right) \\
& \quad=\frac{1}{1+R} \tilde{S}_{t} E_{Q}\left(u^{Z_{t+1}} d^{1-Z_{t+1}} \mid \mathcal{F}_{t}\right) .
\end{aligned}
$$

It follows that a necessary condition is given by

$$
E_{Q}\left(u^{Z_{t+1}} d^{1-Z_{t+1}} \mid \mathcal{F}_{t}\right)=1+R,
$$

hence, taking expectations:

$$
\begin{aligned}
& E_{Q}\left(u^{Z_{t+1}} d^{1-Z_{t+1}}\right) \\
& \quad=u Q\left(Z_{t+1}=1\right)+d Q\left(Z_{t+1}=0\right) \\
& \quad=1+R
\end{aligned}
$$

We get

$$
Q\left(Z_{t+1}=1\right)=1-Q\left(Z_{t+1}=0\right)=q_{u},
$$

where $q_{u}$ is defined as in the Example. But actually more can concluded from formula (4). The conditional expectation $E_{Q}\left(u^{Z_{t+1}} d^{1-Z_{t+1}}\right.$ $\left.\mathcal{F}_{t}\right)$ is equal to $1+R$ on each partition element $\left\{Z_{t}=a_{t}, \ldots, Z_{1}=a_{1}\right\}$, so

$$
\begin{aligned}
& k E_{Q}\left(u^{Z_{t+1}} d^{1-Z_{t+1}} \mid Z_{t}=a_{t}, \ldots, Z_{1}=a_{1}\right) \\
& \quad=u Q\left(Z_{t+1}=1 \mid Z_{t}=a_{t}, \ldots, Z_{1}=a_{1}\right) \\
& \quad+u Q\left(Z_{t+1}=0 \mid Z_{t}=a_{t}, \ldots, Z_{1}=a_{1}\right) \\
& \quad=1+R,
\end{aligned}
$$

and it follows that

$$
\begin{aligned}
& Q \\
& \quad\left(Z_{t+1}=1 \mid Z_{t}=a_{t}, \ldots, Z_{1}=a_{1}\right) \\
& \quad=1-Q\left(Z_{t+1}=0 \mid Z_{t}=a_{t}, \ldots, Z_{1}=a_{1}\right) \\
& \quad=q_{u} .
\end{aligned}
$$

Hence

$$
\begin{aligned}
& Q\left(Z_{t+1}=1, Z_{t}=a_{t}, \ldots, Z_{1}=a_{1}\right) \\
& \quad=Q\left(Z_{t+1}=a_{t+1}\right) Q\left(Z_{t}=a_{t}, \ldots, Z_{1}=a_{1}\right),
\end{aligned}
$$

and by induction we see that the random variables $Z_{1}, \ldots, Z_{T}$ are necessarily independent under $Q$. Summing up, a necessary condition for the existence of an equivalent martingale measure $Q$ is that $0<d<1+R<u$ and the random variables $Z_{1}, \ldots, Z_{T}$ are iid, with distribution $Q\left(Z_{i}=1\right)=1-Q\left(Z_{i}=0\right)=q_{u}$. This condition determines a unique probability $Q$ :

$$
Q(\omega)=q_{u}^{U_{T}(\omega)}\left(1-q_{u}\right)^{T-U_{T}(\omega)} .
$$

It is left to the reader to check that this condition is also sufficient. So for $0<d<1+R<u$ 
there exists a unique martingale measure and we may conclude that the Cox-Ross-Rubinstein model is in this case an arbitrage-free, complete market model. Note that the price $S_{t}$ has a binomial distribution:

$$
\begin{array}{r}
Q\left(S_{t}=s u^{k} d^{t-k}\right)=\left(\begin{array}{l}
t \\
k
\end{array}\right) q_{u}^{k}\left(1-q_{u}\right)^{t-k}, \\
k=0, \ldots, t .
\end{array}
$$

\section{References}

[1] Harrison, J.M. \& Kreps, D.M., "Martingales and Arbitrage in Multiperiod Securities Markets," J. of Economic Theory, 20, (1979), 381-408.

[2] Harrison, J.M. \& Pliska, S.R., "Martingales and Stochastic Integrals in the Theory of Continuous Trading," Stochastic Processes and their Applications, 11, (1981), 215-260.

[3] Koch Medina, P. \& Merino, S., Mathematical Finance and Probability, Birkhäuser, Basel, 2003.

[4] Pliska, S.R., Introduction to Mathematical Finance, Blackwell, Oxford, 1997.

[5] Veal, S.R., Stocks Bonds Options Futures, second edition, New York Institute of Finance, New York, 2001.

\section{Notes}

1) Each share of a stock normally represents a proportional ownership interest in a company.

2) Shorting a stock means selling shares that one does not own, but instead has temporarily borrowed. So one borrows the value of the stock now and pays back the price of the stock at a later time point, that has been agreed on. the interest that has to be paid will therefore depend on the development of the price of the stock. See [5] for more information about financial markets.

3) A collection $\mathcal{F}$ of subsets of $\Omega$ is an algebra if: (i) $\Omega \in \mathcal{F}$, (ii) if $A \in \mathcal{F}$, then $A^{c}=\Omega \backslash A \in \mathcal{F}$, (iii) if $A, B \in \mathcal{F}$, then $A \cup B \in \mathcal{F}$. In the case of infinite sample spaces, one has to consider $\sigma$-algebra's. A collection of events is an $\sigma$-algebra if it is an algebra satisfying property iii': if $A_{1}, A_{2}, \ldots$ is a sequence of events in $\mathcal{F}$, then $\bigcup_{n} A_{n} \in \mathcal{F}$.

4) Remember that $P(A \mid B)=P(A \cap B) / P(B)$ and that $1_{B}$ denotes the indicator of the set $B$, i.e. $1_{B}(\omega)=1$ if $\omega \in B,=0$ otherwise.

5) A random variable $X$ is $\mathcal{F}$-measurable if the sets $\{X \leq a\}, a \in \mathbb{R}$, belong to $\mathcal{F}$. If $\mathcal{F}$ is generated by the partition $\mathcal{P}$, then, $X$ is $\mathcal{F}$ measurable if and only if $X=\sum_{B \in \mathcal{P}} x_{B} 1_{B}$.

6) Two events $A$ and $B$ are independent if $P(A \cap B)=P(A) P(B)$. Two algebra's $\mathcal{F}$ and $\mathcal{G}$ are independent if the events $F$ and $G$ are independent for all choices of $F \in \mathcal{F}$ and $G \in \mathcal{G}$. A random variable $Y$ and an algebra $\mathcal{F}$ are independent if the algebra's $\mathcal{F}_{Y}$ and $\mathcal{F}$ are independent, where $\mathcal{F}_{Y}$ denotes the algebra generated by the partition $\{Y=a\}, a \in Y(\Omega)$.

7) Polya's urn contains $b$ black and $w$ white balls. One draws an arbitrary ball from the urn and replaces it with a new ball of the same colour, and so on. The fraction black balls after the $n$th drawing is given by $Z_{n}=\left(Y_{n}+b\right) /(b+w+n)$, where $Y_{n}$ denotes the number of times up to and including $n$ that a black ball was drawn from the urn. Then $\left(Z_{n}\right)$ is a martingale with respect to its natural filtration. 